\title{
Nowcasting causality in mixed frequency vector autoregressive models
}

Citation for published version (APA):

Götz, T. B., \& Hecq, A. W. (2013). Nowcasting causality in mixed frequency vector autoregressive models. Maastricht University, Graduate School of Business and Economics. GSBE Research Memoranda No. 050 https://doi.org/10.26481/umagsb.2013050

Document status and date:

Published: 01/01/2013

DOI:

10.26481/umagsb.2013050

Document Version:

Publisher's PDF, also known as Version of record

\section{Please check the document version of this publication:}

- A submitted manuscript is the version of the article upon submission and before peer-review. There can be important differences between the submitted version and the official published version of record.

People interested in the research are advised to contact the author for the final version of the publication, or visit the DOI to the publisher's website.

- The final author version and the galley proof are versions of the publication after peer review.

- The final published version features the final layout of the paper including the volume, issue and page numbers.

Link to publication

\footnotetext{
General rights rights.

- You may freely distribute the URL identifying the publication in the public portal. please follow below link for the End User Agreement:

www.umlib.nl/taverne-license

Take down policy

If you believe that this document breaches copyright please contact us at:

repository@maastrichtuniversity.nl

providing details and we will investigate your claim.
}

Copyright and moral rights for the publications made accessible in the public portal are retained by the authors and/or other copyright owners and it is a condition of accessing publications that users recognise and abide by the legal requirements associated with these

- Users may download and print one copy of any publication from the public portal for the purpose of private study or research.

- You may not further distribute the material or use it for any profit-making activity or commercial gain

If the publication is distributed under the terms of Article $25 \mathrm{fa}$ of the Dutch Copyright Act, indicated by the "Taverne" license above, 


\section{Maastricht University}

Thomas Götz, Alain Hecq

Now casting Causality in Mixed

Frequency Vector

Autoregressive Models

RM/13/050

\section{GSBE}

Maastricht University School of Business and Economics

Graduate School of Business and Economics

P.O Box 616

NL- 6200 MD Maastricht

The Netherlands 


\title{
Nowcasting Causality in Mixed Frequency Vector Autoregressive Models
}

\author{
Thomas B. Götz* \\ Alain Hecq \\ Maastricht University, SBE, Department of Quantitative Economics
}

September 3, 2013

\begin{abstract}
This paper introduces the notion of nowcasting causality for mixed-frequency VARs as the mixed-frequency version of instantaneous causality. We analyze the relationship between nowcasting and Granger causality in the mixed-frequency VAR setting of Ghysels (2012) and illustrate that nowcasting causality can have a crucial impact on the significance of contemporaneous or lagged high-frequency variables in standard MIDAS regression models.
\end{abstract}

JEL Codes: C12, C22, C32

JEL Keywords: Instantaneous Causality, Granger Causality, Mixed Frequency VAR, Mixed Data Sampling, MIDAS

\footnotetext{
${ }^{*}$ Corresponding author: Thomas Götz, Department of Quantitative Economics, School of Business and Economics, Maastricht University, P.O. Box 616, 6200 MD Maastricht, The Netherlands. Email: t.gotz@maastrichtuniversity.nl, Tel.: +31 43388 3578, Fax: +31 433882000.
} 


\section{Mixed-frequency VARs}

Economic time series are available at different frequencies. For two variables, ${ }^{1}$ let us denote by $y_{t}, t=1, \ldots, T$ the low-frequency variable and by $x_{t-i / m}^{(m)}$ the high-frequency variables with $m$ high-frequency observations per low-frequency period $t$. The value of $i$ indicates how many high-frequency observations, starting from the end of the low-frequency period, i.e., $i=0$, we take into account. For the quarter/month-scenario $m=3$ and for $t=2012 Q 4, i=1$ refers to November $2012 ; x_{2012 Q 4-2 / 3}^{(3)}$ to October 2012 etc. While it has been the standard approach to address this issue by simply aggregating the high-frequency series, it potentially leads to a loss of information. Mi(xed) Da(ta) S(ampling) regressions (Ghysels et al., 2004) have been developed as means to preserve the information embedded in the higher frequencies without sacrificing parsimony of the model. Until recently, mixed-frequency problems were limited to a simple regression framework, in which one of the low-frequency variables is chosen as the dependent variable. Since the work of Ghysels (2012) for stationary series and the extension of Götz et al. (2013) or Miller and Ghysels (2013) for the non-stationary and possibly cointegrated case, we can analyze the links between high- and low-frequency series in a VAR system treating all variables as endogenous. Letting $X_{t}=\left(x_{t}^{(m)}, x_{t-1 / m}^{(m)}, \ldots, x_{t-(m-1) / m}^{(m)}\right)^{\prime}$, a dynamic structural equations model for $Z_{t}=\left(y_{t}, X_{t}^{\prime}\right)^{\prime}$ is given by $A_{c} Z_{t}=A_{1} Z_{t-1}+\ldots+A_{p} Z_{t-p}+\varepsilon_{t}$, with a diagonal covariance matrix $\Sigma_{\varepsilon}$ and where $A_{c}$ pertains to contemporaneous relationships between the series. $^{2}$ For a quarter/month-example and with $p=1$ the model reads as

$$
\left(\begin{array}{cccc}
1 & \beta_{1} & \beta_{2} & \beta_{3} \\
\delta & 1 & -\rho_{1} & -\rho_{2} \\
0 & 0 & 1 & -\rho_{1} \\
0 & 0 & 0 & 1
\end{array}\right)\left(\begin{array}{c}
y_{t} \\
x_{t}^{(3)} \\
x_{t-1 / 3}^{(3)} \\
x_{t-2 / 3}^{(3)}
\end{array}\right)=\left(\begin{array}{cccc}
\rho_{y} & \phi_{1} & \phi_{2} & \phi_{3} \\
\pi_{1} & \rho_{3} & 0 & 0 \\
\pi_{2} & \rho_{2} & \rho_{3} & 0 \\
\pi_{3} & \rho_{1} & \rho_{2} & \rho_{3}
\end{array}\right)\left(\begin{array}{c}
y_{t-1} \\
x_{t-1}^{(3)} \\
x_{t-1-1 / 3}^{(3)} \\
x_{t-1-2 / 3}^{(3)}
\end{array}\right)+\left(\begin{array}{c}
\varepsilon_{1 t} \\
\varepsilon_{2 t} \\
\varepsilon_{3 t} \\
\varepsilon_{4 t}
\end{array}\right)
$$

where an $\mathrm{AR}(3)$ structure for the high-frequency process is assumed in this example; ${ }^{3}$ the relationship between matrices $A_{c}$ and $A_{1}$ (Ghysels, 2012) is seen in (1). The matrix $A_{c}$ links contemporaneous values of $y$ and $x: \beta_{j} \neq 0$ implies that $y_{t}$ is affected by incoming observations of $X_{t}$, whereas $\delta \neq 0$ implies that the last monthly observation, i.e., March, June and so on, of $x$ is influenced by $y_{t}$. The latter becomes interesting for studying policy analysis, where the high-frequency policy variable may react to current low-frequency conditions. Note that the policy variable could as well be $x_{t-1 / 3}^{(3)}$ or $x_{t-2 / 3}^{(3)}$, although $y_{t}$ may not yet be observed, such that the entire first column of $A_{c}$ is non-zero (see Ghysels, 2012 for details).

\footnotetext{
${ }^{1}$ We can straightforwardly extend the methodology to a multivariate setup as long as we only add lowfrequency variables to the system. As soon as we add further high-frequency variables (with possibly different $m$ ), another framework is required due to the implicit multi-step nature of testing concomitant with the mixedfrequency characteristic of the time series involved. This is, however, left for further research.

${ }^{2}$ The analysis can straightforwardly be adapted to the presence of deterministic terms.

${ }^{3}$ Note that an $\operatorname{AR}(m)$ structure for the high-frequency process is often considered for finance applications, where, e.g., a daily volatility variable may depend on its value on the previous day, during the previous week and the entire previous month (Corsi, 2009).
} 


\section{Nowcasting causality}

Starting from (1) we get the reduced form VAR model $Z_{t}=A_{1}^{*} Z_{t-1}+u_{t}$

$$
Z_{t}=\left(\begin{array}{cccc}
\rho_{y}^{*} & \phi_{1}^{*} & \phi_{2}^{*} & \phi_{3}^{*} \\
\pi_{1}^{*} & a_{2,2}^{*} & a_{2,3}^{*} & a_{2,4}^{*} \\
\pi_{2}^{*} & a_{3,2}^{*} & a_{3,3}^{*} & a_{3,4}^{*} \\
\pi_{3}^{*} & a_{4,2}^{*} & a_{4,3}^{*} & a_{4,4}^{*}
\end{array}\right) Z_{t-1}+u_{t}
$$

where $A_{1}^{*}=A_{c}^{-1} A_{1}$ and $u_{t}=A_{c}^{-1} \varepsilon_{t}$ and $\Sigma_{u}=A_{c}^{-1} \Sigma_{\varepsilon} A_{c}^{-1 \prime}$. Without giving a formal definition of Granger (non-)causality (GC hereafter), it is clear that testing for $X$ not Granger causing $y$ corresponds to $\phi_{1}^{*}=\phi_{2}^{*}=\phi_{3}^{*}=0$ jointly (Ghysels et al., 2013). Importantly, it is defined in terms of the low frequency, i.e., in terms of index $t$. Given the mixed-frequency nature of the variables under consideration, it may be of interest to analyze whether knowing the values of $X_{t}$ helps to predict $y_{t}$. In the common-frequency setup this is referred to as instantaneous causality (Lütkepohl, 2005, p.42), because one tests for a causality pattern between $y_{t}$ and $X_{t}$, where $t$ refers to the common frequency under consideration. Since we intend to predict $y_{t}$ using values of the high-frequency variables within period $t$ (Giannone et al., 2008), we refer to instantaneous causality in the mixed-frequency case as nowcasting causality.

Formally, let $\Omega_{t}$ represents the set of information available at moment $t$ such that $x_{t}^{(m)} \in \Omega_{t}$, but, e.g., $x_{t+1 / m}^{(m)} \notin \Omega_{t}$, and let $\Omega_{t}^{W}$ be the corresponding information set containing the information for all stochastic processes except $W$. Now, we denote by $P\left[y_{t+1} \mid \Omega_{t}^{W}\right]$ and $P\left[X_{t+1} \mid \Omega_{t}^{W}\right]$ the best linear forecasts of $y_{t+1}$ and $X_{t+1}$, respectively, based on $\Omega_{t}^{W}$. We can then define nowcasting causality (NC hereafter) as follows:

Definition 1 y does not nowcasting cause $X$ if

$$
P\left[X_{t+1} \mid \Omega_{t} \cup \Omega_{t+1}^{X}\right]=P\left[X_{t+1} \mid \Omega_{t}\right] .
$$

Similarly, $X$ does not nowcasting cause $y$ if

$$
P\left[y_{t+1} \mid \Omega_{t} \cup \Omega_{t+1}^{y}\right]=P\left[y_{t+1} \mid \Omega_{t}\right] .
$$

In other words, knowing $y_{t+1}$ does not help in predicting $X_{t+1}$ and vice versa.

Considering (1), testing for $\mathrm{NC}$ in both directions corresponds to $\beta_{1}=\beta_{2}=\beta_{3}=0$ and $\delta=0$. Because of the interconnection between the matrices $A_{c}$ and $A_{1}$ the question arises in what sense the presence or absence of NC has an impact on GC. Let us use our quarter/monthexample and consider various specifications of the matrices $A_{c}$ and $A_{1}$ in (1). (i) If $\phi_{j} \neq 0 \forall j$ we will end up with GC in the VAR in (2), no matter whether NC is present or absent. (ii) In the absence of NC, i.e., $\beta_{j}=\delta=0 \forall j$, and if $\phi_{j}=0 \forall j$, then $\phi_{j}^{*}=0 \forall j$ in (2). (iii) Assuming, however, that $\beta_{j} \neq 0$ and $\phi_{j}=0 \forall j$, it turns out that $\phi_{j}^{*} \neq 0 \forall j$ in (2). In other words, the 
presence of NC from $X$ to $y$ implies GC in the same direction. (iv) If $\delta \neq 0$ and $\beta_{j}=0 \forall j$, and if $\phi_{j}=0 \forall j$, then there is no GC from $X$ to $y$ in the reduced-form VAR parameters:

$$
A_{1}^{*}=\left(\begin{array}{cccc}
1 & 0 & 0 & 0 \\
\pi_{1}-\delta+\pi_{2} \rho_{1}+\pi_{3} \widetilde{\rho} & \rho_{3}+\rho_{1} \rho_{2}+\rho_{1} \widetilde{\rho} & \rho_{1} \rho_{3}+\rho_{2} \widetilde{\rho} & \rho_{3} \widetilde{\rho} \\
\phi_{2}+\pi_{3} \rho_{1} & \widetilde{\rho} & \rho_{3}+\rho_{1} \rho_{2} & \rho_{1} \rho_{3} \\
\pi_{3} & \rho_{1} & \rho_{2} & \rho_{3}
\end{array}\right)
$$

where $\widetilde{\rho}=\rho_{1}^{2}+\rho_{2}$.

The matrix $A_{c}$ also determines $\Sigma_{u}=A_{c}^{-1} \Sigma_{\varepsilon} A_{c}^{-1 \prime}$. Actually, in the VAR, $X_{t}$ is not nowcasting causal for $y_{t}$ if and only if the corresponding errors are uncorrelated (Lütkepohl, 2005). In the absence of $\mathrm{NC} \Sigma_{u}$ is indeed block diagonal:

$A_{c}=\left(\begin{array}{cccc}1 & 0 & 0 & 0 \\ 0 & 1 & -\rho_{1} & -\rho_{2} \\ 0 & 0 & 1 & -\rho_{1} \\ 0 & 0 & 0 & 1\end{array}\right) \Rightarrow A_{c}^{-1} \Sigma_{\varepsilon} A_{c}^{-1 \prime}=\left(\begin{array}{cccc}\sigma_{L} & 0 & 0 & 0 \\ 0 & \sigma_{H}\left(1+\rho_{1}^{2}+\widetilde{\rho}^{2}\right) & \rho_{1} \sigma_{H}(1+\widetilde{\rho}) & \sigma_{H} \widetilde{\rho} \\ 0 & \rho_{1} \sigma_{H}(1+\widetilde{\rho}) & \sigma_{H}\left(\rho_{1}^{2}+1\right) & \rho_{1} \sigma_{H} \\ 0 & \sigma_{H} \widetilde{\rho} & \rho_{1} \sigma_{H} & \sigma_{H}\end{array}\right)$,

where $\sigma_{L}$ and $\sigma_{H}$ are the variances of $\varepsilon_{1 t}$ and $\varepsilon_{j t}, j=2,3,4$, respectively, i.e., the entries of the diagonal matrix $\Sigma_{\varepsilon}$.

The presence of NC can be tested using a standard Wald test on the $(1, j)$-elements of $\widehat{\Sigma_{u}}$, i.e., $\widehat{\sigma}_{1, j}=\frac{1}{T} \sum_{t=1}^{T} \widehat{u}_{1 t} \widehat{u}_{j t}, j=2,3,4$, where $\widehat{u}_{j t}$ corresponds to the residual of equation $j$ in the corresponding VAR. Note that the test is asymptotically distributed as $\chi_{m}^{2}$ (see, e.g., Hamilton, 1994, p. 301 or Lütkepohl, 2005, p. 104) and that the concept is fully symmetric in the VAR, i.e., if $y_{t}$ is nowcasting causal for $X_{t}$, then $X_{t}$ is nowcasting causal for $y_{t}$. This implies that in practice we cannot distinguish between $\mathrm{NC}$ in one or the other direction, i.e., whether $\delta \neq 0$ or $\beta_{j} \neq 0$, we can merely check for its presence or absence.

\section{Mixing GC and NC in MIDAS regressions}

Let us now investigate the relationship between the mixed-frequency VAR and univariate MIDAS regressions. Partitioning $\Sigma_{u}$ in the quarter/month VAR(1) example as

$$
\Sigma_{u}=\left(\begin{array}{cc}
\sigma_{1,1} & \sigma_{\cdot 1}^{\prime} \\
\sigma_{\cdot 1} & \Sigma_{2: 4}
\end{array}\right), \text { where } \sigma_{\cdot 1}=\left(\sigma_{2,1}, \sigma_{3,1}, \sigma_{4,1}\right)^{\prime} \text { and } \Sigma_{2: 4}=\left(\begin{array}{ccc}
\sigma_{2,2} & \cdot & \cdot \\
\sigma_{3,2} & \sigma_{3,3} & \cdot \\
\sigma_{4,2} & \sigma_{4,2} & \sigma_{4,4}
\end{array}\right)
$$


we can factorize the VAR in (2) into the conditional model for $y_{t}$ given $X_{t}$ and the remaining marginal models:

$$
\begin{aligned}
y_{t}= & \sigma_{\cdot 1}^{\prime} \Sigma_{2: 4}^{-1} X_{t}+\left[\rho_{y}^{*}-\sigma_{\cdot 1}^{\prime} \Sigma_{2: 4}^{-1}\left(\begin{array}{c}
\pi_{1}^{*} \\
\pi_{2}^{*} \\
\pi_{3}^{*}
\end{array}\right)\right] y_{t-1} \\
+ & {[\underbrace{\left(\begin{array}{c}
\phi_{1}^{*} \\
\phi_{2}^{*} \\
\phi_{3}^{*}
\end{array}\right)}_{\phi^{*}}-\sigma_{\cdot 1}^{\prime} \Sigma_{2: 4}^{-1} \underbrace{\left(\begin{array}{ccc}
a_{2,2}^{*} & a_{2,3}^{*} & a_{2,4}^{*} \\
a_{3,2}^{*} & a_{3,3}^{*} & a_{3,4}^{*} \\
a_{4,2}^{*} & a_{4,3}^{*} & a_{4,4}^{*}
\end{array}\right)}_{A_{2: 4}^{*}}] X_{t-1}+\left[u_{1 t}-\sigma_{\cdot 1}^{\prime} \Sigma_{2: 4}^{-1}\left(\begin{array}{c}
u_{2 t} \\
u_{3 t} \\
u_{4 t}
\end{array}\right)\right] }
\end{aligned}
$$

or

$$
y_{t}=\pi^{*} y_{t-1}+\underbrace{\sum_{i=0}^{2} \theta_{i, 0} x_{t-i / 3}^{(3)}}_{\sigma_{\cdot 1}^{\prime} \Sigma_{2: 4}^{-1} X_{t}}+\underbrace{\sum_{i=0}^{2} \theta_{i, 1} x_{t-1-i / 3}^{(3)}}_{\left(\phi^{*}-\sigma_{\cdot 1}^{\prime} \Sigma_{2: 4}^{-1} A_{2: 4}^{*}\right) X_{t-1}}+u_{t}^{*},
$$

using straightforward substitutions and rearrangements. (4) is a standard mixed-frequency regression, the parameters of which being estimated unrestrictedly (what Foroni et al., 2012 call U-MIDAS) or using MIDAS restrictions (e.g., Ghysels et al., 2004). Equation (4) reveals that with a mixed-frequency VAR as the underlying data generating process, the univariate mixed-frequency model can mix GC and NC.

Indeed, Granger non-causality from $X$ to $y$ in the mixed-frequency VAR implies $\phi^{*}=\mathbf{0}$, whereas nowcasting non-causality corresponds to $\sigma_{\cdot 1}^{\prime}=\mathbf{0}$. Assuming that the VAR in (2), derived from (1), generates the data, let us consider an analyst who works with the mixedfrequency regression model in (4) instead, i.e., who assumes the high-frequency variables $X_{t}$ to be weakly exogenous for the parameters of interest. Three cases, in which the analyst may draw different conclusions from the parameter estimates of (4) than from the ones of (2), can be distinguished:

Case 1: In the absence of GC from $X$ to $y$ in the mixed-frequency VAR (i.e., $\phi^{*}=\mathbf{0}$ ) it is still possible to obtain non-zero coefficients on $X_{t-1}$ in (4), namely if $y$ is nowcasting causal for $X$ (and hence $\sigma_{\cdot 1}^{\prime} \neq \mathbf{0}$ ). This case corresponds to the autoregressive matrix in (3) and is analyzed in detail via Monte Carlo simulations.

Case 2: As indicated above, the parameters in (4) are often estimated after MIDAS restrictions (e.g., Ghysels et al., 2004) have been imposed. Without going into too much detail, instead of estimating all $\theta$-coefficients unrestrictedly, which could lead to parameter proliferation issues (especially for large $m$ ), the polynomial lag structure is hyper-parameterized to yield

$$
y_{t}=\pi^{*} y_{t-1}+\beta \sum_{j=0}^{1} \sum_{i=0}^{2} w_{3 j+i+1}(\gamma) x_{t-j-i / 3}^{(3)}+\epsilon_{t},
$$


where $w(\gamma)$ is a weight function, for which different specifications are proposed in the literature (see, e.g., Ghysels et al., 2007). In the sequel we employ the two-dimensional exponential Almon lag polynomial. Contrary to estimating one weight function for all high-frequency variables as done in (5), one can also estimate a separate weight function for each set of high-frequency variables per $t$-period:

$$
y_{t}=\pi^{*} y_{t-1}+\beta_{1} \sum_{i=0}^{2} w_{i+1}^{1}\left(\gamma^{1}\right) x_{t-i / 3}^{(3)}+\beta_{2} \sum_{i=0}^{2} w_{i+1}^{2}\left(\gamma^{2}\right) x_{t-1-i / 3}^{(3)}+v_{t} .
$$

Let us come back to the estimation of (4) instead of (2). In the absence of NC, but presence of GC from $X$ to $y$, i.e., $\sigma_{.1}^{\prime}=\mathbf{0}$ and $\phi^{*} \neq \mathbf{0}$, (6) is probably more appropriate than (5): Due to nowcasting non-causality, coefficients on $X_{t}$ should equal zero, whereas the ones on the respective observations in period $t-1$ should be non-zero due to existing GC. This implies an abrupt jump in the coefficients from zero to a non-zero value. In (6) this is easily achieved when $\widehat{\beta}_{1}=0$, while (5) probably yields non-zero coefficients for some observations in $X_{t}$. This case is illustrated by means of an application in Section 5 , and in particular Figure 2.

Case 3: Suppose $X$ Granger causes $y$ in the mixed-frequency VAR, i.e., $\phi^{*} \neq \mathbf{0}$, and NC is present as well, i.e., $\sigma_{.1}^{\prime} \neq \mathbf{0}$, with the additional restriction that $\phi^{*}=\sigma_{\cdot 1}^{\prime} \Sigma_{2: 4}^{-1} A_{2: 4}^{*}$. In this unlikely case $\widehat{\theta_{i, 1}}, i=0,1,2$, from (4) could be statistically indistinguishable from zero.

\section{Monte Carlo study}

We analyze Case 1 presented in the previous section by generating the $\operatorname{VAR}(1)$ in (2) from the following dynamic structural equations model:

$$
\left(\begin{array}{cccc}
1 & 0 & 0 & 0 \\
\delta & 1 & -0.4 & 0.2 \\
0 & 0 & 1 & -0.4 \\
0 & 0 & 0 & 1
\end{array}\right)\left(\begin{array}{c}
y_{t} \\
x_{t}^{(3)} \\
x_{t-1 / 3}^{(3)} \\
x_{t-2 / 3}^{(3)}
\end{array}\right)=\left(\begin{array}{cccc}
0.5 & 0 & 0 & 0 \\
0.3 & 0.6 & 0 & 0 \\
0.3 & -0.2 & 0.6 & 0 \\
0.3 & 0.4 & -0.2 & 0.6
\end{array}\right)\left(\begin{array}{c}
y_{t-1} \\
x_{t-1}^{(3)} \\
x_{t-1-1 / 3}^{(3)} \\
x_{t-1-2 / 3}^{(3)}
\end{array}\right)+\left(\begin{array}{c}
\varepsilon_{1 t} \\
\varepsilon_{2 t} \\
\varepsilon_{3 t} \\
\varepsilon_{4 t}
\end{array}\right)
$$

where $\sigma_{L}=\sigma_{H}=1$. Since $\beta_{j}=\phi_{j}=0 \forall j, A_{1}^{*}$ has the same structure as in (3). We test for Granger and nowcasting non-causality in the mixed-frequency VAR using a standard Wald test on $\widehat{\phi_{j}^{*}} \forall j$ for the former and the one described at the end of Section 2 for the latter. Furthermore, we test $\theta_{0,1}=\theta_{1,1}=\theta_{2,1}=0$ in (4), i.e., the significance of $X_{t-1}$ in the univariate mixed-frequency regression. We do so for $\delta \in[-0.95,0.95]$ and $T=50,250$. Figure 1 depicts the various rejection frequencies at a 5\% significance level and are all based on 10,000 replications using GAUSS12.

Due to the absence of GC independent from $\delta$ the corresponding rejection frequencies for $T=250$ almost equal the nominal size of $5 \%$, whereas the test is oversized for $T=50$. For 
Figure 1: Rejection frequencies of the various tests

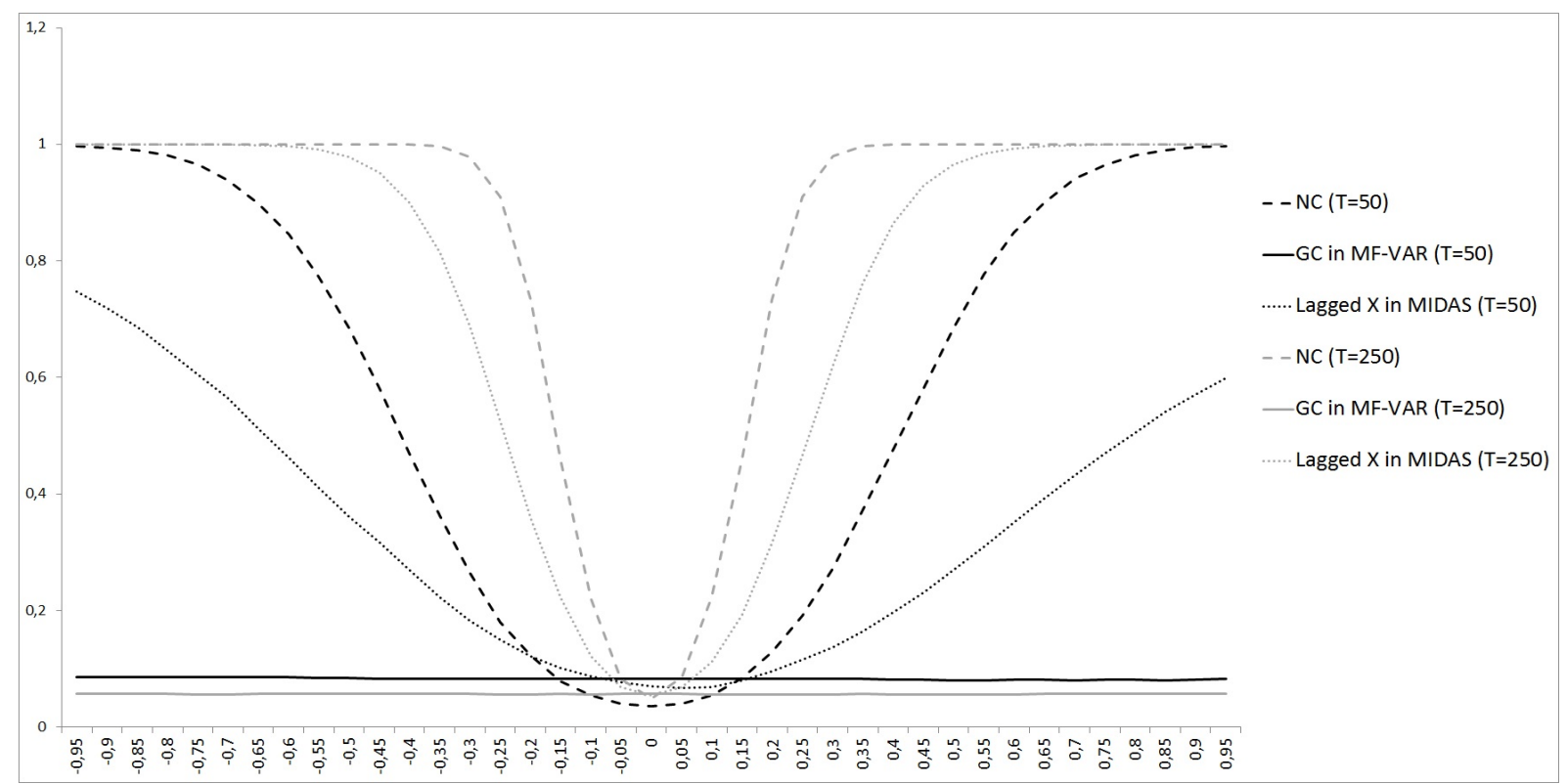

Note: This figure shows the rejection frequencies for Granger non-causality (solid lines) and nowcasting noncausality (dashed lines) in the mixed-frequency VAR as well as for the insignificance of $X_{t-1}$ in the univariate mixed-frequency regression (dotted lines) at the $5 \%$ level. The data are generated from (7) with $\delta \in[-0.95,0.95]$ (horizontal axis). The sample sizes considered are $T=50$ (black lines) and 250 (grey lines). 
$\delta=0$, the test for NC has an actual size almost identical to the nominal one for both sample sizes. The rejection frequencies increase quickly (more rapidly for large $T$ ) as we turn away from the null. As expected, the test for $\theta_{0,1}=\theta_{1,1}=\theta_{2,1}=0$ in (4) behaves similarly to the one for NC. It rejects close to $5 \%$ of the times for $\delta=0$ and more often as $|\delta|$ increases. However, its rejection frequencies are lower than the ones for NC for both sample sizes: From (4), we have that $\sum_{i=0}^{2} \theta_{i, 1} x_{t-1-i / 3}^{(3)}=\left(\phi^{*}-\sigma_{\cdot 1}^{\prime} \Sigma_{2: 4}^{-1} A_{2: 4}^{*}\right) X_{t-1}$, which, for a DGP characterized by an autoregressive matrix as in (3), boils down to $\left(\begin{array}{ccc}\delta \frac{\rho_{3}}{\delta^{2}+1} & 0 & 0\end{array}\right) X_{t-1}$. With $\rho_{3}=0.6$ fixed, we know that for reasonable values ${ }^{4}$ of $\delta$ the coefficient of $x_{t-1}^{(3)}$ is going to be different from zero.

\section{Applications}

This section illustrates the three cases outlined in Section 3 using data from the Federal Reserve Bank of St. Louis. Whenever non-stationarity in the time series is detected, we compute growth rates in order to achieve stationarity. Cointegration issues are neglected here for simplicity, but the methodology can be extended to the non-stationary, possibly cointegrated, case (see Götz et al., 2013).

We first consider the relationship between the monthly growth rate of the seasonally adjusted industrial production index (IPI hereafter), and the weekly growth rate of the seasonally adjusted stock of money M2 (M hereafter). Estimated on the period from January 1991 to December 2010 the mixed-frequency $\operatorname{VAR}(1)$ model with $m=4$ gives

$$
\left(\begin{array}{c}
\widehat{I P I_{t}} \\
\widehat{M_{t}^{(4)}} \\
\widehat{M_{t-1 / 4}^{(4)}} \\
\widehat{M_{t-2 / 4}^{(4)}} \\
\widehat{M_{t-3 / 4}^{(4)}}
\end{array}\right)=\widehat{\mu}+\left(\begin{array}{ccccc}
0.284 & 0.092 & -0.105 & -0.469 & 0.213 \\
(0.069) & (0.205) & (0.194) & (0.266) & (0.182) \\
-0.06 & -0.019 & 0.008 & -0.09 & -0.168 \\
(0.025) & (0.073) & (0.069) & (0.094) & (0.064) \\
-0.021 & 0.153 & 0.017 & -0.014 & 0.021 \\
(0.026) & (0.076) & (0.071) & (0.098) & (0.067) \\
0.013 & 0.137 & -0.044 & -0.014 & 0.077 \\
(0.017) & (0.05) & (0.047) & (0.065) & (0.044) \\
-0.015 & 0.01 & -0.171 & 0.033 & 0.107 \\
(0.025) & (0.073) & (0.069) & (0.095) & (0.065)
\end{array}\right)\left(\begin{array}{c}
I P I_{t-1} \\
M 2_{t-1}^{(4)} \\
M 2_{t-5 / 4}^{(4)} \\
M 2_{t-6 / 4}^{(4)} \\
M 2_{t-7 / 4}^{(4)}
\end{array}\right)
$$

where standard errors are displayed in brackets beneath the least squares coefficient estimates. The Wald test for $H_{0}: \phi_{1,1}=\phi_{1,2}=\phi_{1,3}=\phi_{1,4}=0$, i.e., $\xi_{G C_{-} V A R}$, has a $p$-value of 0.2484 . The Wald statistic for testing nowcasting non-causality à la Hamilton (1994), i.e., $\sigma_{.1}^{\prime}=\mathbf{0}$, turns out to be $\xi_{N C}=36.55$ with a $p$-value of practically $0 \%$. Thus, Granger non-causality from $\mathrm{M}$ to IPI in the mixed-frequency VAR as well as NC between the two series is detected. A mixed-frequency regression in (4) yields:

$$
\begin{aligned}
\widehat{I P I}_{t}= & \underset{(0.0007)}{0.0038}+\underset{(0.065)}{0.196 I P I_{t-1}}-\underset{(0.179)}{1.069} M_{t}^{(4)}-\underset{(0.175)}{0.667} M_{t-1 / 4}^{(4)}+\underset{(0.249)}{0.27} M_{t-2 / 4}^{(4)} \\
& \underset{(0.169)}{0.441} M_{t-3 / 4}^{(4)}+\underset{(0.192)}{0.142} M_{t-1}^{(4)}-\underset{(0.18)}{0.148 M_{t-5 / 4}^{(4)}}-\underset{(0.244)}{0.557 M_{t-6 / 4}^{(4)}}+\underset{(0.171)}{0.073 M_{t-7 / 4}^{(4)}} .
\end{aligned}
$$

\footnotetext{
${ }^{4} \lim _{|\delta| \rightarrow \infty} \delta \frac{\rho_{3}}{\delta^{2}+1}=0$ such that for $\delta$ large enough the corresponding coefficient converges to zero. However, for $|\delta| \leq 5$ we have that $\theta_{0,1}>0.1$.
} 
Now, for testing whether $\theta_{0,1}=\theta_{1,1}=\theta_{2,1}=\theta_{3,1}=0$ the associated Wald test has a $p$-value of 0.0821 . Hence, in the univariate mixed-frequency regression and at the $10 \%$ significance level we would conclude the set of lagged money-variables to be statistically different from zero.

The second pair of data consists of the monthly variation of the seasonally adjusted civilian unemployment rate ( $\mathrm{U}$ hereafter), as regressand, and the aforementioned $\mathrm{M}$, as regressor. The time period considered as well as $m$ are the same as for the first set of data, i.e., $T=240$ and $m=4$. Testing for Granger non-causality in the mixed-frequency $\operatorname{VAR}(1)$ yields a $p$-value equal to 0.004 , testing for nowcasting non-causality gives $p$-value $=0.678$, and checking for non-causality in the mixed-frequency regression results in a $p$-value of 0.0059 . In other words, there is no NC between the two series, but M continues to be statistically significant when conditioning on the high-frequency variables.

Here it is of interest to analyze what happens if MIDAS restrictions are imposed and, in particular, if we estimate only one or two weight functions, i.e., estimate (5) or (6). Given the estimates of $\gamma=\left(\gamma_{1}, \gamma_{2}\right)^{\prime}$ in (5) with $m=4$, we can compute the corresponding aggregate version of $\mathrm{M}$ as $\sum_{j=0}^{1} \sum_{i=0}^{3} w_{4 j+i+1}(\widehat{\gamma}) M_{t-j-i / 4}^{(4)}$. This allows us to run a least squares regression and compute the standard error of the scale coefficient $\widehat{\beta}=31.05$ as well as its $t$-statistic. ${ }^{5}$ With $t_{\widehat{\beta}}=4.01$ the corresponding coefficients on the whole set of $\mathrm{M}$ variables $(t$ and $t-1)$ are jointly statistically different from zero. These coefficients can be obtained by multiplying the $\gamma$ dependent weights by the scale coefficient and are plotted as solid line in Figure 2. Crucially and contrary to the missing NC feature, some $M_{t}$-variables possess a non-zero coefficient $\left(M_{t-3 / 4}^{(4)}\right.$ and to a lesser extent $M_{t-2 / 4}^{(4)}$ ). If, however, (6) with $m=4$ is estimated, $t_{\widehat{\beta_{1}}}=1.34$ and $t_{\widehat{\beta_{2}}}=3.84$ implying that $\widehat{\beta_{1}}$, corresponding to the instantaneous $\mathrm{M}$ variables, is not significantly different from zero. In other words, the nowcasting non-causality feature is preserved when estimating two separate weight functions. This is reflected by the associated coefficients plotted as dotted line in Figure 2.

In the third application, the dependent variable is the quarterly growth rate of the seasonally adjusted real gross national product (GNP hereafter); the growth rate of IPI is the independent variable such that $m=3$. This pair of time series is observed from 1948Q3 to 2011Q4. After estimating a $\operatorname{VAR}(1)$ we find that $\xi_{G C_{-} V A R}=105.8$ leading to a clear rejection of the associated null hypothesis. Testing for nowcasting non-causality yields $\xi_{N C}=78.18$ such that both GC in the mixed-frequency VAR and NC are detected. Estimation of the univariate mixed-frequency regression leads to

$$
\begin{aligned}
\widehat{G N P}_{t}= & \underset{(0.0005)}{0.0053}-\underset{(0.064)}{0.12} G N P_{t-1}+\underset{(0.04)}{0.211 I P I_{t}^{(3)}}-\underset{(0.062)}{0.045 I P I_{t-1 / 3}^{(3)}}+\underset{(0.047)}{0.253 I P I_{t-2 / 3}^{(3)}} \\
& +\underset{(0.06)}{0.042 I P I_{t-1}^{(3)}}-\underset{(0.062)}{0.067 I P I_{t-4 / 3}^{(3)}}+\underset{(0.039)}{0.07} I P I_{t-5 / 3}^{(3)} .
\end{aligned}
$$

Testing for $\theta_{0,1}=\theta_{1,1}=\theta_{2,1}=0$, however, amounts to a $p$-value of 0.2997. Consequently,

\footnotetext{
${ }^{5}$ Due to non-identification of the weight-specifying parameter $\gamma$ under the null hypothesis, we would have to follow the approach of Davies (1987) and compute Hansen (1996)'s p-value. Although not presented and reported here, the results do not differ qualitatively. The same holds for the estimation of (6).
} 
Figure 2: Coefficients on $M_{t}$ and $M_{t-1}$ with one or two separate weight functions

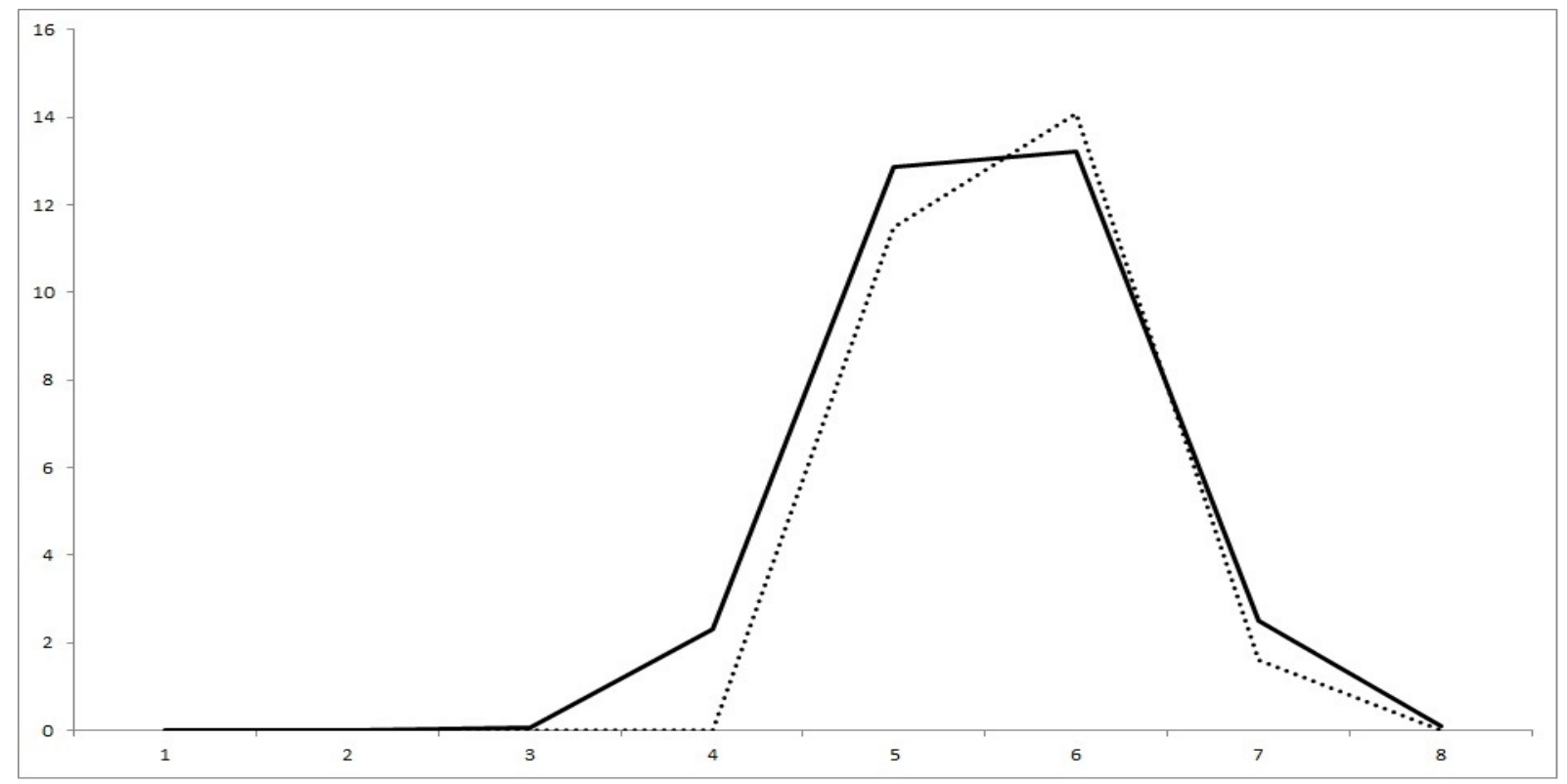

Note: This figure shows the coefficients, i.e., their respective weights $\times$ scale coefficient, of $M_{t}^{(4)}, \ldots, M_{t-3 / 4}^{(4)}$ and $M_{t-1}^{(4)}, \ldots, M_{t-7 / 4}^{(4)}$, once when only one function is computed on the whole set of variables (solid line) and once when two separate weight functions, one for each set of 4 variables (dotted line), are computed. The weight functions are obtained using the two-dimensional Almon Lag Polynomial (see, e.g., Ghysels et al., 2007). The estimates are $\widehat{\beta}=31.05, \widehat{\gamma}=(9.34,-0.75)^{\prime}$ for the solid line and $\left(\widehat{\beta_{1}}, \widehat{\beta_{2}}\right)^{\prime}=(12.6,27.2)^{\prime}, \widehat{\gamma^{1}}=$ $(1.4,-0.27)^{\prime}$ and $\widehat{\gamma^{2}}=(3.75,-1.18)^{\prime}$ for the dotted line. Due to the fact that the scale coefficient corresponding to $M_{t}^{(4)}, \ldots, M_{t-3 / 4}^{(4)}$, i.e., $\widehat{\beta_{1}}$, is statistically not different from zero, its estimate is set to zero. 
estimating the univariate mixed-frequency regression instead of the mixed-frequency VAR leads to $I P I_{t-1}$ being statistically insignificant.

\section{Acknowledgements}

We thank an anonymous referee, Michael Eichler, Daniela Osterrieder and Jean-Pierre Urbain for useful suggestions and comments on earlier versions of the paper. 


\section{References}

Corsi, F. (2009). A simple approximate long-memory model of realized volatility. Journal of Financial Econometrics, 7(2):174-196.

Davies, R. B. (1987). Hypothesis testing when a nuisance parameter is present only under the alternatives. Biometrika, 74(1):pp. 33-43.

Foroni, C., Marcellino, M., and Schumacher, C. (2012). U-midas: Midas regressions with unrestricted lag polynomials. CEPR Discussion Papers 8828, C.E.P.R. Discussion Papers.

Ghysels, E. (2012). Macroeconomics and the reality of mixed frequency data. Working paper, University of North Carolina.

Ghysels, E., Motegi, K., and Hill, J. (2013). Granger causality tests with mixed data frequencies. Discussion Paper.

Ghysels, E., Santa-Clara, P., and Valkanov, R. (2004). The midas touch: Mixed data sampling regression models. CIRANO Working Papers 2004s-20, CIRANO.

Ghysels, E., Sinko, A., and Valkanov, R. (2007). Midas regressions: Further results and new directions. Econometric Reviews, 26(1):53-90.

Giannone, D., Reichlin, L., and Small, D. (2008). Nowcasting: The real-time informational content of macroeconomic data. Journal of Monetary Economics, 55(4):665-676.

Götz, T. B., Hecq, A., and Urbain, J.-P. (2013). Testing for common cycles in non-stationary vars with varied frecquency data. Advances in Econometrics Vol. 31, forthcoming.

Hamilton, J. D. (1994). Time series analysis. Princeton Univ. Press, Princeton, NJ.

Hansen, B. E. (1996). Inference when a nuisance parameter is not identified under the null hypothesis. Econometrica, 64(2):413-30.

Lütkepohl, H. (2005). New introduction to multiple time series analysis. Springer Publishing Company, Incorporated.

Miller, J. I. and Ghysels, E. (2013). Testing for cointegration with temporally aggregated and mixed-frequency time series. Working paper, University of Missouri. 